www.jmscr.igmpublication.org

Impact Factor 5.84

Index Copernicus Value: 71.58

ISSN (e)-2347-176x ISSN (p) 2455-0450

crossref DOI:_https://dx.doi.org/10.18535/jmscr/v5i9.30

\title{
Secondary Hydatidosis- A Surgical Challenge
}

\author{
Authors

\section{Girish D Bakhshi, Rajesh G Chincholkar, Jasmine R Agarwal, Madhukar R Gupta,} \\ Prachiti S Gokhe, Vinay Prasad \\ Department of General Surgery, Grant Government Medical College and Sir J. J. Group of Hospitals, \\ Mumbai, India \\ Corresponding Author \\ Dr Girish D. Bakhshi \\ Devneeti, Plot-61, Sector-7, Koper Khairane, Navi Mumbai-400709, Maharashtra, India \\ Email: gdbakhshi@yahoo.com, Phone: +919820218198
}

\begin{abstract}
Introduction: Hydatid cyst, a common parasitic infection caused by Echinococcus granulosus, may get disseminated intra-abdominally after rupture of a hydatid cyst in the peritoneal cavity, producing secondary echinococcosis.

Case Description: A 49 years old male with generalised abdominal pain and low-grade fever. He was previously operated in 2013 for hepatic hydatid cyst from a midline laparotomy. Presently he was diagnosed with recurrence of liver hydatid disease with secondary peritoneal hydatidosis after detailed clinical and radiological evaluation. After starting oral Albendazole, open marsupialization of the liver hydatid cyst was donewith complete excision of multiple peritoneal hydatid cysts. Albendazole was continued for 3 months postoperatively and the patient was found to be disease-free in the 6-month follow up period.

Discussion: Peritoneal hydatidosis may be either primary or secondary. The secondary form is often due to spillage during surgery or rupture of a hepatic hydatid cyst. Peritoneal echinococcosis typically remains silent for years and the symptoms are polymorphous, depending on the location of the cyst.Ultrasonography and computed tomography are both excellent imaging modalities for the diagnosis. The treatment of choice for localized peritoneal cyst is principally a careful and complete surgical excision after isolation with scolicidal soaked mops. Among non-operative options are chemotherapy and percutaneous treatment, depending on the features of the cysts.

Conclusion: Surgery for primary hydatid disease should be meticulously done, taking care to avoid spillage of scolices in the peritoneal cavity by isolating the cyst using Savlon soaked gauze and patient should be started on oral Albendazole therapy to prevent recurrence of hydatid cyst and secondary peritoneal echinococcosis.
\end{abstract}

\section{INTRODUCTION}

Hydatid disease is a common parasitic infection caused by Echinococcus granulosus, where humans are the accidental intermediate host by ingestion of eggs. Common sites include liver $(75 \%)$ and lung $(15 \%)^{1}$. Disseminated intraabdominal hydatid disease $(13 \%)$ may occur following a rupture of the hydatid cyst into the 
peritoneal cavity producing secondary echinococcosis. Depending on the location of the cyst, a patient may present with varied symptoms and signs.

\section{CASE DESCRIPTION}

A 49 years old male patient presented with an 8day history of generalized pain in abdomen and intermittent episodes of low-grade fever. He did not have any other complaints. He was previously operated for a hepatic hydatid cyst in 2013, when an open hydatid cyst excision was done through a midline laparotomy.

Clinical examination revealed soft and non-tender abdomen with a globular lump palpable in the left hypochondrium, measuring $4 \times 4 \quad \mathrm{~cm}$ approximately.

His routine hematological and serological investigations were within normal limits.

An ultrasonography (USG) of the abdomen revealed multiple well-defined loculated cystic lesions in the epigastrium, left hypochondrium and pelvis, most likely hydatid cysts.

Contrast enhanced computerised tomographic (CECT) study of the abdomen and pelvis was done for a detailed evaluation. It showed a large well-defined thin walled exophytic peripherally enhancing hypodense lesion in the left lobe of liver measuring $13.4 \times 5.9 \times 7.1 \mathrm{~cm}$ having multiple thin septae and multiple thin peripheral incomplete calcifications along its wall. It also showed multiple small internal cysts within suggestive of daughter cysts. Adjacent fat planes were well maintained. 5 other similar morphology lesions were seen in the mesentry in the gastrohepatic region (measuring $5.2 \times 5.4 \times 4.7 \mathrm{~cm}$ ), on the left side abutting the jejunal loops (measuring $5.4 \times 5.9 \times 6.5 \mathrm{~cm}$ ) and 3 in the lower abdomen abutting the rectosigmoid colon, urinary bladder and distal ileal loops (measuring $5.6 \times 4.2 \times 5.5 \mathrm{~cm}$, $6.9 \times 5.3 \times 5.4 \mathrm{~cm}$ and $8.3 \times 5.9 \times 7.3 \mathrm{~cm}$ ) [Fig 1]. The above findings were suggestive of a liver hydatid disease with peritoneal hydatidosis.

The patient was started on oral Albendazole 10 $\mathrm{mg} / \mathrm{kg}$, after which he was planned for, exploratory laparotomy. Intraoperative findings revealed multiple intra-abdominal cysts with a large left lobe hepatic hydatid cyst. Open marsupialization of the liver hydatid cyst was donewith complete excision of multiple peritoneal hydatid cysts [Fig 2]. The hepatic hydatid cyst cavity was aspirated carefully after instillation of scolicidal agent, avoiding spillage in the peritoneal cavity.

Postoperative period was uneventful. The patient was discharged on postoperative day 10 on course of oral Albendazole. Follow up of 6 months has shown him to be disease and symptom-free.



Figure 1: CECT showing hepatic hydatid cyst in the left lobe with multiple intraperitoneal hydatid cysts 


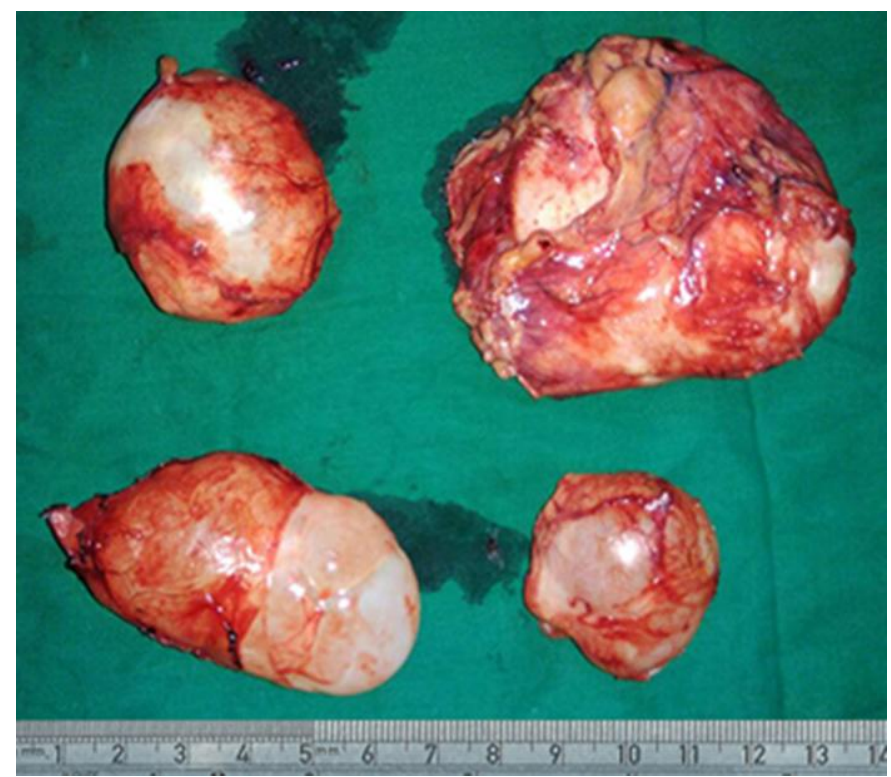

Figure 2: Excised specimens of multiple intraperitoneal hydatid cysts

\section{DISCUSSION}

Peritoneal hydatidosis may be either primary or secondary. The primary form is less common, and is either hematogenous or heterotopic by block migration of a hepatic hydatid cyst, which has ruptured its adventitious and kept its membrane intact ${ }^{2}$. The secondary form is often due to cracking or rupture of a hepatic hydatid cyst, or less commonly splenic hydatid cyst. The rupture of the primary hydatid cyst may occur spontaneously, favored by the large size of the cyst. The rupture can also occur during an effort by increasing the intra-abdominal pressure or after trauma. One of the common causes of secondary peritoneal hydatidosis is spillage of contents from a previously operated primary hydatid cyst while attempting its surgical excision. In present case, patient was operated for hepatic hydatid cyst 4 years back, and had now presented with secondary cysts in abdomen.

Peritoneal echinococcosis typically remains silent for years and is usually undetected unless cysts are large enough to cause symptoms. Even then, the symptoms are polymorphous and depend on the location of the cyst ${ }^{3}$. The diagnosis is usually not a problem. Ultrasonography and computed tomography are both excellent imaging modalities for the detection of hydatid cysts. CECT, however, is superior to the former for providing accurate topographic information ${ }^{2}$.

The treatment of choice for localized peritoneal cyst is principally a careful and complete surgical excision ${ }^{4}$. The pathway must achieve proper cyst exposure, allow easy abdominal exploration and treat associated lesions. Among non-operative options are chemotherapy and percutaneous treatment. Chemotherapy with oral Albendazole $10 \mathrm{mg} / \mathrm{kg} /$ dayis effective in small thin walled cysts with diameter less than $4 \mathrm{~cm}$. It is suitable in patients with high risk for surgery, and in patients with multiple peritoneal cysts and cysts in bone/ brain/ multiple organs. It also prevents secondary echinococcosis after spillage of scolices in peritoneal cavity effectively, when given for 3 months in both preoperative and postoperative periods ${ }^{5}$. Albendazole also acts as a concomitant therapy with percutaneous drainage. Percutaneous USG-guided puncture-aspiration-injectionreaspiration (PAIR) therapy is an effective minimally invasive treatment for Gharbi type I-III hepatic hydatid cysts ${ }^{6,7}$. In this procedure, transhepatic puncture of hydatid cyst is done under USG guidance, followed by serial aspiration of contents, instillation of scolicidal agent and reaspiration of cyst cavity. However, in present case, the multiple peritoneal hydatid cysts were inaccessible for puncture, which is why PAIR therapy was unsuitable in this patient.

In present case, midline exploratory laparotomy was done, followed by excision of all the peritoneal cysts and marsupialization of the liver hydatidcyst after thorough irrigation of the cyst cavity with scolicidal agent and complete aspiration of the cyst contents, avoiding spillage in the peritoneal cavity. Afterwards, the patient was started on oral Albendazole therapyto prevent emergence of recurrent peritoneal hydatidosis ${ }^{5}$.

\section{CONCLUSION}

As secondary peritoneal hydatidosis is usually associated with a previous surgical excision of a primary hydatid cyst, the initial surgery should be meticulously done. Scolicidal agent should be 
adequately used and spillage of cyst contents in the abdominal cavity should be avoideds. Recurrence rates are significantly reduced when chemotherapy with Albendazole is added as an adjunct to the surgical excision. The secondary surgery is planned considering the number and location of the recurrent cysts. These are carefully excised completely whilst taking the abovementioned precautions to prevent subsequent recurrence of the disease.

\section{REFERENCES}

1. Sarkar D, Ray S, Saha M. Peritoneal hydatidosis: A rare form of a common disease. Trop Parasitol. 2011;1(2):123125. doi:10.4103/2229-5070.86962.

2. Daali M, Hssaida R, Zoubir M, Hda A, Hajji A. [Peritoneal hydatidosis: a study of 25 cases in Morocco]. Sante. 10(4):255260.

http://www.ncbi.nlm.nih.gov/pubmed/111 11243. Accessed May 27, 2017.

3. Wani I, Lone AM, Hussain I, Malik A, Thoker M, Wani KA. Peritoneal hydatidosis in a young girl. Ghana Med J. 2010;44(4):163-164.

http://www.ncbi.nlm.nih.gov/pubmed/214 16052. Accessed May 27, 2017.

4. Dziri C, Haouet K, Fingerhut A, Zaouche A. Management of Cystic Echinococcosis Complications and Dissemination: Where is the Evidence? World J Surg. 2009;33 (6):1266-1273. doi:10.1007/s00268-0099982-9.

5. Shams-Ul-Bari, Arif SH, Malik AA, Khaja AR, Dass TA, Naikoo ZA. Role of albendazole in the management of hydatid cyst liver. Saudi J Gastroenterol. 2011;17 (5):343-347. doi:10.4103/13193767.84493 .

6. Gharbi HA, Hassine W, Brauner MW, Dupuch K. Ultrasound examination of the hydatic liver. Radiology. 1981;139(2):459463.

doi:10.1148/radiology.139.2.7220891.
7. Rajesh R, Dalip DS, Anupam J, Jaisiram A. Effectiveness of puncture-aspirationinjection-reaspiration in the treatment of hepatic hydatid cysts. Iran $J$ Radiol. 2013;10(2):68-73.

doi:10.5812/iranjradiol.7370. 